\title{
Propiedades morfológicas de los suelos asociadas a los ecosistemas de Páramo, Nariño, Sur de Colombia
}

\author{
Soil morphological properties related to Páramo ecosystems in Nariño, Southern Colombia
}

\author{
Felipe Zúñiga ${ }^{1,2,3 \ddagger}$, Jenny Huertas ${ }^{4}$, Gabriela Guerrero ${ }^{4}$, Jairo Sarasty ${ }^{5}$, \\ José Dörner ${ }^{2,3}$ y Hernán Burbano Orjuela ${ }^{6}$
}

\footnotetext{
${ }^{1}$ Escuela de Graduados, Facultad de Ciencias Agrarias, Universidad Austral de Chile. Independencia 641, Valdivia, Chile.

*Autor responsable (felipezunigaugalde@gmail.com)

${ }^{2}$ Instituto de Ingeniería Agraria y Suelos, Facultad de Ciencias Agrarias, Universidad Austral de Chile. Valdivia, Chile.

${ }^{3}$ Centro de Investigación en Suelos Volcánicos (CISVo), Universidad Austral de Chile. Valdivia, Chile.

${ }^{4}$ Programa de Ingeniería Ambiental, Facultad de Ingeniería, Universidad Mariana. Calle 18 No. 34-104. San Juan de Pasto, Nariño, Colombia.

${ }^{5}$ Facultad de Ciencias Agrícolas, Universidad de Nariño. Calle 18 Cr 50 Ciudadela Universitaria Torobajo. San Juan de Pasto, Nariño, Colombia.

${ }^{6}$ Centro de Investigación en Ciencias Agrarias, Facultad de Ciencias Agrícolas, Universidad de Nariño, Torobajo, San Juan de Pasto, Colombia.
}

\section{RESUMEN}

Franklin Delano Roosevelt (1882-1945) indicó: "Una nación que destruye su suelo, se destruye a sí misma". ¿Cómo podemos acercar el suelo a la sociedad para que esta sentencia no se convierta en una realidad? Una alternativa es desarrollar herramientas didácticas que permitan socializar la información que los perfiles de suelo nos pueden entregar. Se estudiaron propiedades morfológicas de los suelos asociados a los ecosistemas de Páramos en Nariño, Sur de Colombia y su vínculo con los servicios ecosistémicos (SE) a través de una función de pedotransferencia (FPTs); con un enfoque instructivo, orientado a la regulación ambiental de la zona de estudio. Se describieron morfológicamente 42 horizontes de suelo en un rango de nueve ecosistemas (páramos hasta cultivos) en la zona Andina de Nariño, Sur de Colombia. En general, los suelos más profundos se encontraron en sistemas de páramos y bosque nativo, presentaron una coloración más oscura, evidencia de mayor acumulación de carbono orgánico en el suelo. La densidad aparente del suelo varío entre 0.13 a $1.35 \mathrm{Mg} \mathrm{m}^{-3}$ y el carbono orgánico del suelo entre 0.60 a $23.77 \%$ Esta propiedad explicó la densidad aparente del suelo $\left(P<0.0001 ; \mathrm{r}^{2}=0.83\right)$ y se describió a partir de una ecuación inversa de primer orden. El carbono orgánico del suelo es un indicador útil para estimar aspectos cuantitativos del sistema poroso del suelo que permiten inferir servicios ecosistémicos de regulación

Cita recomendada:

Zúñiga, F., J. Huertas, G. Guerrero, J. Sarasty, J. Dörner y H. Burbano Orjuela. 2018. Propiedades morfológicas de los suelos asociadas a los ecosistemas de Páramo, Nariño, Sur de Colombia. Terra Latinoamericana 36: 183-196. DOI: https://doi.org/10.28940/terra.v36i2.363 de agua, aire y nutrientes de los Páramos. Finalmente, se propusieron "cartillas informativas de suelo" como herramientas que permitirán informar y crear conciencia en los pobladores de la zona de influencia respecto de la importancia del suelo como capital natural y evitar que la sentencia de F.D. Rossevelt se convierta en una incómoda realidad.

Palabras clave: usos de suelo, función de pedotransferencia, cartillas de suelo, Andisol.

\section{SUMMARY}

Franklin Delano Roosevelt (1882-1945) stated: “A nation that destroys its soils destroys itself." How can we get the soil closer to society so that this sentence does not become reality? One alternative is to develop didactic tools that allow socializing the information that soil profiles can provide. Soil morphological properties associated with páramo ecosystems and their link with ecosystem services (SE) were studied in Nariño, Southern Colombia through a pedotransference function (FPTs) with an instructive approach, oriented to the environmental regulation of the study area. Fortytwo soil horizons were described morphologically in a range of 9 ecosystems (páramos to crops). In general, the deepest soils are found in páramos and native forest systems. These soils are also dark, evidence of accumulation of soil organic carbon. Soil bulk density 
varied between 0.13 and $1.35 \mathrm{Mg} \mathrm{m}^{-3}$, and soil organic carbon ranged between 0.60 and $23.77 \%$. This property explains soil bulk density $\left(P<0.0001 ; \mathrm{r}^{2}=0.83\right)$ and is described by an inverse first order equation. Soil organic carbon is a useful indicator to estimate quantitative aspects of the soil pore system and to infer the ecosystem services of water, air and nutrient regulation of the páramos. Finally, "Land information cards" are proposed as tools to will inform and raise awareness of the society regarding the importance of the soil as natural capital and prevent the sentence of F.D. Roosevelt from becoming an uncomfortable reality.

Index words: land uses, pedotransfer soil function, soil cards, Andisols.

\section{INTRODUCCIÓN}

El suelo y la humanidad se vinculan íntimamente. Desde el desarrollo de las primeras civilizaciones (Mesopotamia, sumerios y egipcios), el suelo y sus propiedades han tenido un rol fundamental en la selección de sitios para el establecimiento de los pueblos (Brevik y Hartemink, 2010). Este desarrollo ha estado potenciado por herramientas tecnológicas que han permitido p. ej. labrar el suelo, como el arado (Pryor, 1985); no obstante, Leonardo da Vinci hace más de 600 años indicaba que "Sabemos más acerca de los cuerpos celestes que del suelo bajo nuestros pies", haciendo referencia acerca del escaso conocimiento que, en esa era, existía del suelo. Actualmente, sabemos mucho más acerca de nuestros suelos; de su historia (Krupenikov, 1993; Brevik y Hartemink, 2010), de sus procesos pedológicos (Jenny, 1941; Simonson, 1959), de su taxonomía (Hartemink y Bockheim, 2013; WRB, 2014; Soil Survey Staff, 2014), de su mineralogía (Besoain, 1985), de su biodiversidad (Brussaard et al., 2007), de su relación con el cambio climático y la seguridad alimentaria (Lal, 2004; Burbano-Orjuela, 2016) y de los servicios ecosistémicos que nos proveen (MEA, 2005; Dominati et al., 2010; Adhikari y Hartemink, 2016).

A pesar de la proclamación realizada el 20 de diciembre de 2013 en Nueva York por la Asamblea General de las Naciones Unidas (UN, 2013), en donde se declaró el 05 de diciembre como el día Mundial del Suelo y de la proclamación del 07 de diciembre de 2015 en Viena realizada por la Unión Internacional de la Ciencia del Suelo (IUSS, 2015) donde se declaró la década Internacional de los Suelos (2015-2024), existe aún una desconexión entre el conocimiento que se tiene de los suelos y las necesidades de la sociedad. Esto conlleva que las decisiones políticas, sociales y económicas que se toman en torno a problemáticas ligadas a la ciencia del suelo sean incongruentes.

\section{EI Suelo, su Investigación y la Sociedad}

El suelo es parte de la biósfera, y se relaciona directamente con el paisaje, la vegetación, el clima y la sociedad en su conjunto. El suelo como tal ha sido definido de múltiples formas de acuerdo con las disciplinas de estudio (Bockheim et al., 2005). Si bien el suelo es un cuerpo que puede ser evaluado a través de los sentidos, resulta complejo imaginar su funcionamiento interno. En general, el suelo se estudia como un cuerpo aislado en donde evaluamos sus propiedades físicas, químicas y biológicas entre otras, que nos permiten aceptar, rechazar y generar nuevas hipótesis asociadas a su estudio.

Estas hipótesis buscan -o al menos deberíanresolver problemáticas que aquejan a nuestra sociedad, más aún si consideramos que los recursos que financian nuestras investigaciones son escasos y provienen de los impuestos que los contribuyentes entregan a la administración estatal.

Como producto de nuestros trabajos surgen artículos de investigación originales (AIO). Esta práctica es necesaria para avanzar en el conocimiento, pues esa masa crítica -que en ocasiones puede ser reducida- es la revisora y lectora de estos AIO, sin embargo, también es la herramienta de evaluación de nuestros investigadores frente a las instituciones de los Estados que suministran el financiamiento a los proyectos de investigación. Estos documentos entregan información relevante y pertinente para la comunidad científica. Sin embargo, esta información no siempre se encuentra depurada para el resto de las personas que componen una sociedad, por ello la comprensión de las ideas y conclusiones principales de los proyectos de investigación se reducen a uno o más AIO.

Por lo anterior, para nosotros, investigadores vigentes en las Ciencias Naturales resulta un desafío mantenernos vigentes en nuestro quehacer, pero a la vez, es esencial transmitir esta información de la manera más clara y didáctica posible, de manera tal, que la sociedad en su conjunto pueda comprender y 
visualizar -en este caso- al suelo como un componente dinámico de la biósfera y no como el concepto de "tierra" -que en ocasiones suena despreciativo- y que básicamente alude al suelo como un sustrato que nos entrega los alimentos y no considera otros servicios ecosistémicos (SE), sociales y culturales que este componente de la biósfera proporciona. En ese contexto, Zúñiga et al. (2014a) plantean que antes de seleccionar y destinar el suelo para cualquier uso o fin (agronómico, forestal, conservación etc.), es necesario plantearse las siguientes inquietudes: i) ¿Puedo hacer uso de este suelo para determinado fin? ii) ¿Cuáles son las mayores limitantes que el suelo presenta? iii) ¿Es el uso más adecuado el que se le otorgará al suelo? iv) ¿Qué información se puede obtener con solo observar el perfil? v) ¿Qué servicios ecosistémicos este suelo puede proveer?

\section{El Suelo y los Servicios Ecosistémicos (SE)}

Los SE corresponden a los bienes y servicios que los ecosistemas proveen a la sociedad (MEA, 2005). Estos beneficios se encuentran directa o indirectamente ligados a las funciones que los ecosistemas realizan (Costanza et al., 1997) y se agrupan en cuatro categorías: i) servicios de provisión (alimentación, fibra, combustible), ii) servicios de regulación (regulación climática, calidad del agua y del aire), iii) servicios culturales (diversidad, cultura, religión) y iv) servicios de soporte (formación de suelo, fotosíntesis) (MEA, 2005).

El concepto de servicio ecosistémico (Figura 1) está vinculado con el concepto de "capital natural", que hace referencia a la existencia de activos naturales (p. ej. suelo) que producen o producirán un flujo de bienes o servicios valiosos para el bienestar humano (Costanza et al., 1997; Dominati et al., 2010).

Las funciones y servicios ecosistémicos que el capital natural suelo provee a la sociedad han sido descritas por diversos autores. Dominati et al. (2010) proponen un marco de referencia más amplio y holístico para comprender los SE. Este marco se compone de: i) el suelo y sus propiedades (inherentes y dinámicas); ii) los procesos involucrados en su formación, degradación y mantención; iii) los "drivers" externos (naturales y antrópicos); iv) los SE culturales, de regulación, provisión, soporte y v) el bienestar humano.

Los trabajos publicados por Costanza et al. (1997) y por MEA (2005), han sido los promotores de nuevos estudios que vinculen las propiedades y funciones del suelo con los SE. Estos estudios se han enfocado principalmente en los servicios de provisión y regulación. No obstante, en Latinoamérica estos estudios no superan el $6 \%$ de los trabajos publicados en el ámbito mundial (Adhikari y Hartemink, 2016).

\section{Los SE Vinculados al Suelo en Colombia}

Colombia es un país excepcionalmente rico en servicios ecosistémicos. Costanza et al. (1997), indicaban que, para gran parte de Colombia, los SE fueron valorados entre US\$2000 a $3000 \mathrm{ha}^{-1} \mathrm{año}^{-1}$. WAVES (2016), indica que el capital natural de este país representa una proporción significativa de la riqueza global per cápita, y por lo tanto, la valoración y gestión

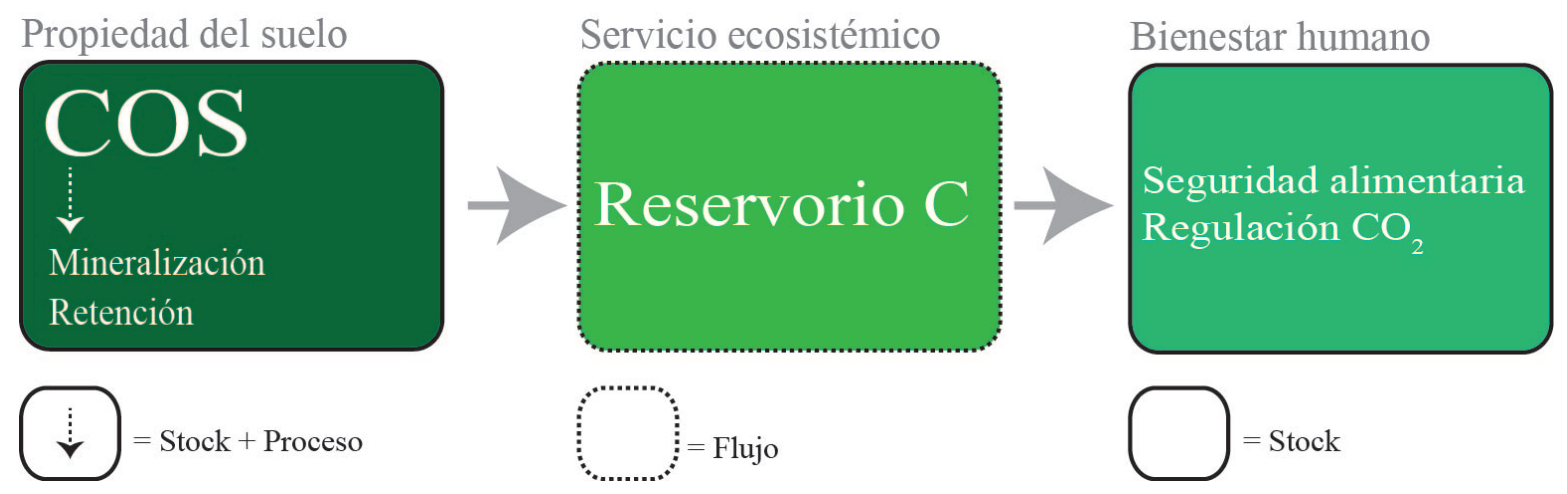

Figura 1. Esquematización del proceso de vinculación entre el suelo, el servicio ecosistémico y el bienestar humano. Los cuadros con líneas continuas indican "stock" de C del suelo. La flecha con línea punteada al interior del cuadro representa los procesos que afectan al $\mathrm{C}$ del suelo. Finalmente, la línea punteada que rodea el cuadro corresponde a los flujos de $\mathrm{C}$ en el suelo. 
adecuada de los recursos naturales se ha convertido en prioridades para el país. Esto se ha visto reflejado en el desarrollo de una "Política para la Gestión Sostenible del Suelo" (GIAS) (MINAMBIENTE, 2016). El objetivo de esta política es "Promover la gestión sostenible del suelo en Colombia, en un contexto integral en el que confluyan la conservación de la biodiversidad, el agua y el aire, el ordenamiento del territorio y la gestión de riesgo, contribuyendo al desarrollo sostenible y al bienestar de los colombianos". Esta política presenta un marco de desarrollo sólido que comprende fases de: i) diagnóstico, ii) planes de acción para el desarrollo sostenible del suelo y iii) seguimiento y evaluación entre otros. Este tipo de políticas debe ser necesariamente una puerta para el desarrollo a largo plazo de una ley del suelo que busque no sólo hacer un mejor uso de este capital natural, sino que sea protegido, regulado y legislado con la importancia que este capital natural merece.

Si bien este tipo de iniciativas son avances que permiten gestionar integralmente el uso y los servicios ecosistémicos que los suelos proveen, es necesario que

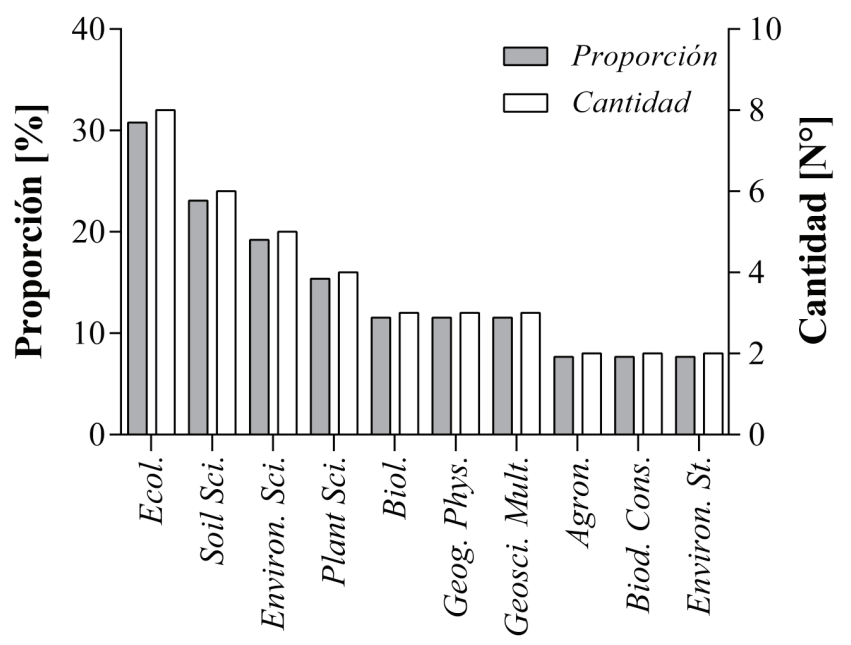

Categorías Web of Science

Figura 2. Categorización de artículos científicos publicados en la base de datos "Web of Science" que consideran servicios ecosistémicos del suelo en Colombia. Ecol. = Ecology; Soil Sci. $=$ Soil Science; Environ. Sci. $=$ Environmental Science; Plant Sci. $=$ Plant Science; Biol. = Biology; Geog. Phys. = Geography Physical; Geosci. Mult. = Geosciences Multidisciplinary; Agron. = Agronomy; Biod. Cons. = Biodiversity Conservation; Environ. St. $=$ Environmental Studies. esto también se refleje en las publicaciones científicas que aborden esta temática.

En una búsqueda temática realizada en la colección principal de "Web of Science" combinando las palabras clave (en inglés) suelos, servicios ecosistémicos y Colombia, se obtuvieron sólo 26 artículos, de los cuales $8(30.8 \%)$ son categorizados en Ecología, 6 (23.1\%) en Ciencias del Suelo y $5(19.2 \%)$ en Ciencias Ambientales (Figura 2).

Engeneral, los SE de los agroecosistemas se vinculan con el contenido de carbono del suelo. Bajo diferentes usos de los suelos volcánicos (bosque nativo, praderas naturales y cultivos forrajeros), Ordoñez et al. (2015), determinaron que el contenido total de $\mathrm{C}$ en el suelo incrementa bajo praderas naturales, promoviendo cambios positivos en propiedades químicas y la actividad microbial del suelo, lo que sugiere que los manejos agrícolas realizados por los campesinos y comunidades indígenas de la zona favorecen el SE de provisión de alimentos. Similares tendencias han sido encontradas por Lavelle et al. (2014), en suelos oxisoles de los Llanos Orientales al este de Colombia, en donde determinaron que el $\mathrm{C}$ del suelo en praderas mejoradas favoreció a las comunidades de macro invertebrados y consecuentemente los $\mathrm{SE}$ de regulación hídrica y biodiversidad. No obstante, no existen estudios en la literatura que vinculen o infieran los SE del suelo a través de sus propiedades morfológicas. Una de las formas de vincular estos bienes y servicios con un perfil de suelo es a través de una función de pedotransferencia (FPTs). Casanova et al. (2003); indican que estas FPTs corresponden a asociaciones o relaciones empíricas entre propiedades fácilmente medibles y parámetros hidráulicos del suelo. No obstante, estas propiedades (textura, densidad aparente y COS) si bien se relacionan con la estructura porosa en un sentido amplio, no caracterizan la funcionalidad del sistema poroso (Pachepsky et al., 2006); es decir solo describen parámetros de capacidad del volumen disponible para el almacenamiento de agua/aire en el suelo (Horn y Kutilek, 2009).

Por lo anteriormente expuesto, la presente investigación tuvo como objetivo el estudio de algunas propiedades morfológicas de los suelos asociados a los ecosistemas de Páramos en Nariño, Sur de Colombia y su vínculo con los SE a través de una FPTs; con un enfoque instructivo, orientado a la regulación ambiental de la zona de estudio. 


\section{MATERIALES Y MÉTODOS}

\section{Zona de Estudio}

Se describieron nueve pedones de suelo distribuidos en tres áreas de estudio (Figura 3) dominados por suelos de origen volcánico en ambientes de páramo, bosque, praderas y cultivo.

Los suelos estudiados corresponden a: i) un transecto de cuatro pedones en las inmediaciones del Volcán Cumbal, tomando como referencia la altitud (m), su uso actual y especies vegetales, ii) tres pedones en la Granja Experimental Botana de la Universidad de Nariño, en donde los usos actuales fueron bosque, pradera y cultivo de papa, iii) los últimos dos pedones se describieron en la zona de El Encano (Reserva Natural "Encanto Andino"), en donde se encontró un ecosistema denominado páramos azonales los cuales se caracterizan por estar sobre los $2800 \mathrm{~m} \mathrm{s.} \mathrm{n.} \mathrm{m.,} \mathrm{los} \mathrm{dos}$ sitios de estudio se diferenciaron por una sucesión de cambio de uso de suelo de páramo propiamente dicho a una pradera naturalizada. El detalle de los sitios de estudio se presenta en el Cuadro 1.

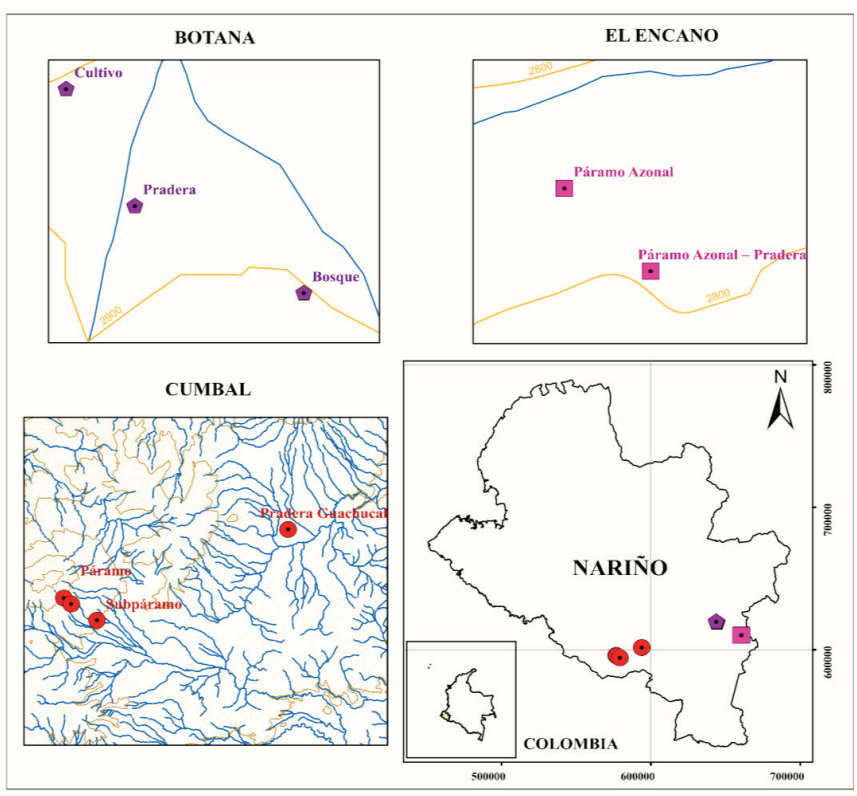

Figura 3. Distribución espacial de los sitios de descripción. Pentágonos púrpura corresponden a los sitios de muestreo en Botana, cuadros fucsias a los sitios en El Encano y círculos rojos a los sitios descritos en Cumbal y Guachucal.

Cuadro 1. Descripción general de los sitios de muestreo.

\begin{tabular}{|c|c|c|c|c|c|}
\hline \multirow{2}{*}{ Sitio } & \multicolumn{2}{|c|}{ Coordenadas } & \multirow{2}{*}{ Altitud } & \multirow{2}{*}{ Uso actual } & \multirow{2}{*}{ Especies vegetales dominantes } \\
\hline & Latitud & Longitud & & & \\
\hline & $-\cdots-\cdots$ & $\circ \ldots \ldots$ & $\mathrm{m}$ & & \\
\hline \multicolumn{6}{|c|}{ Cumbal } \\
\hline & 0.941468 & -77.877990 & 3749 & Súper Páramo (SUP) & Pajonales (Festuca breviaristata Pilg.) \\
\hline & 0.937219 & -77.872962 & 3622 & Páramo (PAR) & Frailejón (Espeletia), pajonales ( $F$. breviaristata) \\
\hline & 0.925494 & -77.855300 & 3402 & Subpáramo - Cultivo (C-PAR) & $\begin{array}{l}\text { Frailejón (Espeletia), pajonales ( } F \text {. breviaristata), } \\
\text { cultivo de papa (Solanum tuberosum) }\end{array}$ \\
\hline & 0.990798 & -77.724220 & 3030 & Pradera Guachucal (PRA-G) & $\begin{array}{l}\text { Holcus lanatus L. Anthoxanthum odoratum L., } \\
\text { Trifolium repens L. y Lolium perenne L. }\end{array}$ \\
\hline \multicolumn{6}{|c|}{ El Encano } \\
\hline & 1.071564 & -77.127285 & 2672 & Páramo Azonal (PAR-AZ) & $\begin{array}{l}\text { Frailejón (Espeletia), pajonales ( } F \text {. breviaristata), } \\
\text { helechos (Blechnum) }\end{array}$ \\
\hline & 1.070398 & -77.126127 & 2805 & Páramo Azonal - Pradera (PRA-AZ) & $\begin{array}{l}\text { A. odoratum, Hypochaeris radicata L., Taraxacum } \\
\text { officinale (L.) Weber ex F. H. Wigg., H. lanatus, } \\
\text { totorilla (Juncus effusus). }\end{array}$ \\
\hline \multicolumn{6}{|c|}{ Botana } \\
\hline & 1.153330 & -77.271667 & 2901 & Bosque (B-BOT) & Bosque seco montano \\
\hline & 1.155583 & -77.275815 & 2841 & Pradera (PRA-BOT) & H. lanatus, A. odoratum, Agrostis capillaris L. \\
\hline & 1.158611 & -77.277500 & 2815 & Cultivo (C-BOT) & Cultivo de papa (S. tuberosum), post-cosecha. \\
\hline
\end{tabular}




\section{Descripción Morfológica}

En cada uno de los lugares de estudio se realizaron calicatas de aproximadamente $1 \mathrm{~m}^{3}$ de volumen con profundidad variable ${ }^{1}$. Se seleccionaron los sitios de muestreo de acuerdo con la vegetación predominante y las variaciones altitudinales de los ecosistemas. Se separaron in situ los horizontes de suelo de acuerdo con los cambios en su coloración y su clase textural. Posteriormente, se determinó la profundidad efectiva del suelo y el espesor de cada horizonte detectado. Utilizando la metodología propuesta por Schoeneberger et al. (2012) se determinaron las siguientes propiedades morfológicas del suelo: i) Color (Munsell soil color book), ii) clase textural (al tacto) iii) estructura (forma, tamaño y grado), iv) consistencia en húmedo y en saturado, v) tamaño y cantidad de raíces, vi) forma y nitidez de los límites de los horizontes y aspectos específicos observables en los horizontes (p. ej. rasgos redoximórficos).

\section{Análisis de Laboratorio}

De cada horizonte de suelo descrito, se determinó el contenido de carbono orgánico total (COT) a partir del método de pérdidas por ignición descrito por Sadzawka et al. (2006), en donde se asumió que la materia orgánica del suelo se compone de un 58\% de C (De Vos et al., 2005). Además, se determinó la densidad aparente del suelo (Dap) a través del método del cilindro (Grossman y Reinsch, 2002).

\section{RESULTADOS Y DISCUSIÓN}

\section{Propiedades Morfológicas de los Suelos}

Los suelos estudiados presentan un rango de variación de profundidad efectiva entre 60 y $150 \mathrm{~cm}$. Los suelos de SUP, PAR-AZ y PRA-BOT, son los que presentaron las menores profundidades, mientras que los suelos de B-BOT, PAR y PRA-AZ presentaron un mayor espacio para la exploración radical en profundidad variando entre $150,110 \mathrm{y}$ $107 \mathrm{~cm}$, respectivamente (Cuadros 2A, 2B y 2C). Esta propiedad determina la capacidad de almacenamiento de agua, así como el espacio que las raíces pueden explorar (Canadell et al., 1996; FAO, 2017a). Hirzel y Matus (2013), indican que la profundidad del suelo determina significativamente $(P<0.01)$ el número de tallos $\mathrm{m}^{-2}$, la altura de plantas y el rendimiento de granos en dos cultivares de trigo en un Andisol del centro sur de Chile. De acuerdo con los criterios establecidos por USDA (1961) y SAG (2016), los suelos descritos en esta investigación varían de moderadamente profundos $(50-90 \mathrm{~cm})$ a muy profundos $(>150 \mathrm{~cm})$, lo que sugiere que desde un punto de vista agrícola o forestal no tendrían limitaciones en su uso. Sin embargo, el que un suelo no posea limitaciones en función de su profundidad, no significa que puede o deba ser utilizado intensivamente. Lal (2014), indica que una disminución en la profundidad del suelo refleja una disminución en su calidad, y consecuentemente, en una reducción de sus funciones y servicios ecosistémicos. Por lo anterior, las decisiones del uso del suelo deben integrar otras propiedades del mismo, como textura, posición topográfica y variación espacial (Heimsath et al., 1999).

No se manifiesta una relación directa entre el incremento en la intensidad de uso del suelo y la disminución de profundidad efectiva. La condición de SUP es la que presenta la menor profundidad en el sitio de Cumbal, (Cuadro 2A) y PAR-AZ en el sitio de El Encano (Cuadro 2B). Sin embargo, en el sitio Botana, los suelos bajo pradera y cultivo ( $>$ intensidad de uso) presentaron los perfiles del suelo más delgados $(60 \mathrm{~cm})$.

Las clases texturales predominantes en este estudio fueron franco limosas (23.8\%), limosas (19\%) y francas (19\%). Las clases texturales franco arcillo arenosas (11.9\%) y arcillo limosa (7.1\%) se asociaron a suelos con pradera y cultivo en Botana, mientras que las clases con predominio de sensación arenosa se asociaron a los suelos de páramo y pradera en el sector de Cumbal y Guachucal, respectivamente, representando un 19\% del total de las clases texturales determinadas en terreno (Figura 4).

De los suelos descritos en este trabajo, la clase textural predominante (franco limosa), ocurre con mayor frecuencia en los horizontes superficiales de todos los sitios evaluados, a excepción de PRA y $\mathrm{C}$ de Botana. Esta clase textural, es característica de suelos derivados de materiales volcánicos (Shoji et al., 1993) que le otorga excelentes propiedades físicas (mecánicas e hidráulicas) a este tipo de suelos.

En los horizontes inferiores de SUP, PAR, y PRA-G, incrementa la sensación arenosa. Este incremento se asocia, posiblemente, al material parental (rocas

${ }^{1}$ La profundidad de la calicata varío de acuerdo con la presencia de factores que limitaran su profundidad efectiva (p. ej. Nivel freático superficial). 
Cuadro 2A. Descripción morfológica de suelos para los sitios de Cumbal.

\begin{tabular}{|c|c|c|c|c|c|c|c|c|c|}
\hline \multirow{2}{*}{ Sitio } & \multirow{2}{*}{$\begin{array}{l}\text { Uso } \\
\text { actual }\end{array}$} & \multirow{2}{*}{ Profundidad } & \multirow{2}{*}{$\mathrm{Hz} .^{\dagger}$} & \multirow{2}{*}{ Textura } & \multirow{2}{*}{ Color } & & \multicolumn{2}{|c|}{ Consistencia } & \multirow{2}{*}{ Comentarios } \\
\hline & & & & & & & Humedad\$ & Saturación & \\
\hline & & $\mathrm{cm}$ & & & & & & & \\
\hline \multicolumn{10}{|c|}{ Cumbal } \\
\hline & SUP & $5-0$ & Oe & n.d & n.d & & n.d & n.d & \\
\hline & & $0-13$ & $\mathrm{OA}$ & $\mathrm{F}$ & $\begin{array}{l}7.5 \mathrm{YR} \\
2.5 / 1\end{array}$ & $\bullet$ & Fri & MP, LAd & \\
\hline & & $13-23$ & $\mathrm{~A} / \mathrm{C}$ & FL & $10 Y R 3 / 2$ & $\bullet$ & Fir & P, LAd & Gravas 20\% \\
\hline & & $23-35$ & $\mathrm{C}$ & $\operatorname{ArF}$ & $10 Y R 4 / 3$ & $\bullet$ & Fir & LP, LAd & $\begin{array}{l}\text { Presencia de franjas de hierro, } \\
\text { gravas } 15 \%\end{array}$ \\
\hline & & $35-60$ & $\mathrm{Cr}$ & $\mathrm{Ar}$ & $10 Y R 5 / 2$ & $\bullet$ & Fir & LAd & Sin estructura (grano simple) \\
\hline & PAR & $5-0$ & $\mathrm{Oa}$ & n.d & n.d & & n.d & n.d & \\
\hline & & $0-33$ & $\mathrm{OA}$ & $\mathrm{L}$ & $10 Y R 2 / 1$ & $\bullet$ & Fri & MP, LAd & \\
\hline & & $33-63$ & A & FL & $10 Y R 3 / 1$ & $\bullet$ & Fir & $\mathrm{P}, \mathrm{Ad}$ & Casts del lombrices \\
\hline & & $63-92$ & $\mathrm{Bw}$ & $\operatorname{ArF}$ & $10 Y R 3 / 1$ & • & Fir & LP, LAd & \\
\hline & & $92-110$ & $2 \mathrm{Ab}$ & FAr & $10 Y R 2 / 1$ & • & Fri & P, LAd & \\
\hline & C-PAR & $0-10$ & Op & $\mathrm{L}$ & $10 \mathrm{YR} 2 / 1$ & $\bullet$ & MFri & LP, LAd & \\
\hline & & $10-13$ & Oi & n.d & n.d & $n . d$ & n.d & n.d & $\begin{array}{l}\text { Horizonte orgánico (cobertura } \\
\text { vegetal dominada por musgos) }\end{array}$ \\
\hline & & $13-20$ & OA1 & $\mathrm{L}$ & $10 Y R 2 / 1$ & $\bullet$ & Fri & MP, Ad & \\
\hline & & $20-55$ & OA2 & $\mathrm{FL}$ & $10 Y R 2 / 1$ & $\bullet$ & MFri & MP, Ad & \\
\hline & & $55-80$ & A1 & $\mathrm{FL}$ & $10 Y R 2 / 1$ & • & Fir & P, LAd & \\
\hline & & $>80$ & A2 & FAr & $10 Y R 2 / 1$ & $\bullet$ & Fir & LP, LAd & \\
\hline & PRA-G & $5-0$ & Oe & n.d & n.d & n.d & n.d & $n . d$ & \\
\hline & & $0-10$ & $\mathrm{OA}$ & $\mathrm{L}$ & $10 Y R 2 / 1$ & $\bullet$ & Fri & P, LAd & $\begin{array}{l}\text { Evidente actividad biológica, } \\
\text { larvas de coleópteros }\end{array}$ \\
\hline & & $10-24$ & A & $\mathrm{F}$ & $10 \mathrm{YR} 2 / 1$ & - & Fri & $\mathrm{P}, \mathrm{Ad}$ & $\begin{array}{l}\text { Rasgos redoximórficos }(2.5 \mathrm{YR} \\
3 / 6) \bullet\end{array}$ \\
\hline & & $24-44$ & $\mathrm{AC}$ & FL & 10YR 2/1 & • & Fir & $\mathrm{P}, \mathrm{Ad}$ & $\begin{array}{l}\text { Rasgos redoximórficos }(2.5 \mathrm{YR} \\
3 / 6) \bullet\end{array}$ \\
\hline & & $44-59$ & $\mathrm{C}$ & $\mathrm{Ar}$ & 10YR 5/1 & - & Fri & LP, LAd & $\begin{array}{l}\text { Rasgos redoximórficos (10YR } \\
5 / 6) \bullet\end{array}$ \\
\hline & & $59-70$ & $2 \mathrm{Ab}$ & FAr & 10YR 2/1 & $\bullet$ & MFir & $\mathrm{P}, \mathrm{Ad}$ & $\begin{array}{l}\text { Sin estructura (masiva), } \\
\text { horizonte saturado de agua }\end{array}$ \\
\hline
\end{tabular}

${ }^{\dagger}$ Corresponde a la denominación de horizontes genéticos maestros. ${ }^{\ddagger}$ Clase textural al tacto. $\mathrm{Ar}=$ arenosa; $\mathrm{ArF}=$ areno francosa; $\mathrm{F}=\mathrm{franca} ; \mathrm{FL}=\mathrm{franco} \mathrm{limosa;}$ $\mathrm{FAr}=$ franco arenosa; $\mathrm{FAL}=$ franco arcillo limosa; $\mathrm{L}=$ limo; $\mathrm{AL}=$ arcillo limosa; n.d $=$ no determinado. ${ }^{\S}$ Determinada según contenido de agua en campo al momento de la descripción del perfil. Fri = friable; Fir = firme; Mfri = muy friable; Mfir = muy firme. " Determinada en condiciones de completa saturación. $\mathrm{MP}=$ muy plástico; $\mathrm{P}=$ plástico; $\mathrm{LP}=$ ligeramente plástico; LAd = ligeramente adhesivo; $\mathrm{Ad}=$ adhesivo.

basálticas) y al bajo desarrollo pedológico que ocurre en profundidad. Bajo PAR-AZ predomina la sensación limosa lo que se vincula con la distribución del material orgánico en el perfil. A su vez, bajo PRA-BOT y C-BOT, predomina la sensación arcillosa, lo que se vincula con la presencia de un horizonte argílico $(\mathrm{Bt})$, es decir, ocurre un proceso de iluviación (migración) de arcilla al interior del perfil del suelo.

La coloración gris parduzca predominó en el perfil descrito en SUP. Por su parte en los usos de suelo PAR y PAR-AZ el color dominante, en gran parte del perfil, fue el negro, lo que también se reflejó en los horizontes 
Cuadro 2B. Descripción morfológica de suelos para los sitios de Eı Encano.

\begin{tabular}{|c|c|c|c|c|c|c|c|c|c|}
\hline \multirow{2}{*}{ Sitio } & \multirow{2}{*}{$\begin{array}{l}\text { Uso } \\
\text { actual }\end{array}$} & \multirow{2}{*}{ Profundidad } & \multirow{2}{*}{$\mathrm{Hz} .^{\dagger}$} & \multirow{2}{*}{ Textura } & \multirow{2}{*}{ Color } & & \multicolumn{2}{|c|}{ Consistencia } & \multirow{2}{*}{ Comentarios } \\
\hline & & & & & & & Humedad $^{\S}$ & Saturación & \\
\hline & & $\mathrm{cm}$ & & & & & & & \\
\hline \multicolumn{10}{|c|}{ El Encano } \\
\hline & PAR-AZ & $0-10$ & Oa1 & n.d & $10 Y R 2 / 1$ & $\bullet$ & n.d & MP, LAd & Horizonte orgánico \\
\hline & & $10-35$ & $\mathrm{Oa} 2$ & $\mathrm{~L}$ & 10YR 2/1 & $\bullet$ & n.d & n.d & Sin estructura (masiva) \\
\hline & & $35-50$ & $\mathrm{Oa} 3$ & FL & 10YR 2/1 & • & n.d & MP, Ad & Sin estructura (masiva) \\
\hline & & $50-60$ & $\mathrm{Oa} 4$ & $\mathrm{~L}$ & $10 Y R 2 / 1$ & $\bullet$ & n.d & MP, LAd & Sin estructura (masiva) \\
\hline & & $>60$ & Oa5 & $\mathrm{L}$ & 10YR 2/1 & $\bullet$ & n.d & P, Ad & $\begin{array}{l}\text { Sin estructura (masiva). Presencia } \\
\text { de napa freática }\end{array}$ \\
\hline & \multirow[t]{7}{*}{ PAR-AZ } & $0-8$ & $\mathrm{Oa}$ & $\mathrm{F}$ & $10 \mathrm{YR} 2 / 1$ & $\bullet$ & Fir & LP, LAd & \\
\hline & & $8-20$ & Ap & $\mathrm{L}$ & $10 Y R 2 / 1$ & $\bullet$ & Fir & P, LAd & \\
\hline & & $20-42$ & $\mathrm{Bw}$ & FL & $10 \mathrm{YR} 2 / 2$ & $\bullet$ & Fir & MP, Ad & $\begin{array}{l}\text { Rasgos redoximórficos }(5 \mathrm{YR} \\
3 / 4) \bullet\end{array}$ \\
\hline & & $42-87$ & $2 \mathrm{Ab}$ & $\mathrm{F}$ & $10 \mathrm{YR} 2 / 1$ & $\bullet$ & Fir & MP, Ad & $\begin{array}{l}\text { Horizonte de transición }(40-60 \mathrm{~cm}) \text {. } \\
\text { Presencia de rasgos redoximórficos }\end{array}$ \\
\hline & & & & & & & & & $\begin{array}{l}\text { y raíces oxidadas }(5 \mathrm{YR} 5 / 8) \bullet \text { al } \\
\text { interior de los agregados }\end{array}$ \\
\hline & & $87-105$ & Bs & $\mathrm{F}$ & 10YR 5/6 & $\bullet$ & Fir & MP, Ad & $\begin{array}{l}\text { Horizonte plácico (Bsm) 5YR 5/6 } \\
\text { - con franja de Mn (2.5YR 2.5/1) } \\
\text { - Presencia de carbón }\end{array}$ \\
\hline & & $105-107$ & $\mathrm{C}$ & Ar & $10 \mathrm{YR} 3 / 3$ & $\bullet$ & n.d & $n . d$ & $\begin{array}{l}\text { Matriz arenosa }(10 \mathrm{YR} 3 / 3) \bullet \mathrm{con} \\
\text { moteados }(2.5 \mathrm{YR} 4 / 6) \bullet\end{array}$ \\
\hline
\end{tabular}

${ }^{\dagger}$ Corresponde a la denominación de horizontes genéticos maestros. ${ }^{\ddagger}$ Clase textural al tacto. $\mathrm{Ar}=$ arenosa; $\mathrm{ArF}=$ areno francosa; $\mathrm{F}=\mathrm{franca} ; \mathrm{FL}=$ franco limosa; $\mathrm{FAr}=$ franco arenosa; $\mathrm{FAL}=$ franco arcillo limosa; $\mathrm{L}=$ limo; $\mathrm{AL}=$ arcillo limosa; $n . d=$ no determinado. ${ }^{\S}$ Determinada según contenido de agua en campo al momento de la descripción del perfil. Fri = friable; Fir = firme; Mfri = muy friable; Mfir = muy firme. " Determinada en condiciones de completa saturación. MP = muy plástico; $\mathrm{P}=$ plástico; $\mathrm{LP}=$ ligeramente plástico; $\mathrm{LAd}=$ ligeramente adhesivo; $\mathrm{Ad}=$ adhesivo.

superficiales de C-PAR, PRA-G y PRA-AZ. Bajo el suelo de bosque predomina la coloración parda en el matiz 7.5YR, mientras que en los sitios de pradera y cultivo en Botana se aprecian coloraciones con tonalidades oliva a partir de los $30 \mathrm{~cm}$ de profundidad (Cuadro 2C).

Las coloraciones predominantemente oscuras (10YR 2/1) encontradas en este trabajo, indican que el comportamiento de los suelos bajo condiciones de páramo y páramos azonales depende de propiedades cuantitativas y cualitativas de la materia orgánica (Ellies et al., 2005). Si bien la materia orgánica participa activamente de los procesos de estabilidad estructural en los suelos (Zúñiga et al., 2014b), en profundidad tiende a disminuir. Esto no solo se refleja en los cambios de coloración del suelo (Cuadro 2), sino que sugiere que el comportamiento de los suelos en profundidad depende de otros factores, tales como la cantidad y tipo de arcilla, así como la presencia de "moteados" (principalmente óxidos de hierro) que participan como agentes cementantes de la estabilización estructural (Zúñiga et al., 2014b), lo que se reflejará en los servicios ecosistémicos que los suelos proveen.

\section{Función de Pedotransferencia y Servicios Ecosistémicos Asociados}

Los servicios ecosistémicos corresponden a los bienes o servicios que el capital natural (en este caso el suelo) provee a la sociedad (MEA, 2005; Dominati et al., 2010). De acuerdo con las propiedades morfológicas evaluadas en este trabajo, se puede inferir que cada horizonte de suelo está proporcionando uno o más servicios ecosistémicos en función de la propiedad de suelo más dominante: 
Cuadro 2C. Descripción morfológica de suelos para los sitios de Botana.

\begin{tabular}{|c|c|c|c|c|c|c|c|c|c|}
\hline \multirow{2}{*}{ Sitio } & \multirow{2}{*}{ Uso actual } & \multirow{2}{*}{ Profundidad } & \multirow{2}{*}{$\mathrm{Hz} .^{\dagger}$} & \multirow{2}{*}{ Textura } & \multirow{2}{*}{ Color } & & \multicolumn{2}{|c|}{ Consistencia } & \multirow{2}{*}{ Comentarios } \\
\hline & & & & & & & Humedad & Saturación & \\
\hline & & $\mathrm{cm}$ & & & & & & & \\
\hline \multicolumn{10}{|c|}{ Botana } \\
\hline & \multirow[t]{7}{*}{ B-BOT } & $5-0$ & Oi & n.d & n.d & & n.d & n.d & Hojarasca de bosque \\
\hline & & $0-17$ & $\mathrm{Oe}$ & n.d & 7.5YR $2.5 / 2$ & $\bullet$ & n.d & n.d & Horizonte orgánico \\
\hline & & $17-28$ & A & $\mathrm{F}$ & 7.5 YR $2.5 / 3$ & $\bullet$ & Fri & LP, Lad & \\
\hline & & $28-70$ & BA & FL & $7.5 Y R 3 / 4$ & $\bullet$ & Fir & $\mathrm{P}, \mathrm{Ad}$ & \\
\hline & & $70-119$ & Bw1 & FL & 10YR 3/6 & $\bullet$ & Fri & $\mathrm{P}, \mathrm{Ad}$ & \\
\hline & & $119-150$ & Bw2 & FL & $7.5 Y R 4 / 6$ & $\bullet$ & Fir & P, Ad & $\begin{array}{l}\text { Concreciones de } \mathrm{Fe} 40 \% \text { ( } 2.5 \mathrm{YR} \\
3 / 4) \bullet\end{array}$ \\
\hline & & $>150$ & $\mathrm{BC}$ & $\mathrm{F}$ & 7.5YR 4/6 & $\bullet$ & Fri & $\mathrm{P}, \mathrm{Ad}$ & \\
\hline & \multirow[t]{4}{*}{ PRA-BOT } & $0-19$ & Ap & F & 10YR $3 / 3$ & $\bullet$ & Fir & P, Ad & $\begin{array}{l}\text { Rasgos redoximórficos }(7.5 \mathrm{YR} \\
5 / 8) \bullet \text {, presencia de cuarzo, } \\
\text { arcilla calcinada y roca } \\
\text { meteorizada }\end{array}$ \\
\hline & & $19-33$ & BAt & FAL & 7.5 YR 5/4 & $\bullet$ & Fir & MP & $\begin{array}{l}\text { Evidencia de actividad biológica, } \\
\text { presencia de carbón }\end{array}$ \\
\hline & & $33-60$ & $\mathrm{Bt}$ & $\mathrm{AL}$ & $5 Y 4 / 2$ & $\bullet$ & Fir & $\mathrm{P}, \mathrm{Ad}$ & Sin estructura (masiva) \\
\hline & & $>60$ & $\mathrm{BCr}$ & $\mathrm{AL}$ & $10 Y R 4 / 3$ & $\bullet$ & Fir & P, Ad & $\begin{array}{l}\text { Rasgos redoximórficos }(2.5 \mathrm{YR} \\
3 / 6) \bullet \text {, presencia de carbón } \\
(70 \mathrm{~cm}) \text {, franja de Fe. Sin } \\
\text { estructura (masiva) }\end{array}$ \\
\hline & \multirow[t]{5}{*}{ C-BOT } & $0-9$ & Ap & FAL & 10YR $2 / 2$ & $\bullet$ & Fir & P, Ad & $\begin{array}{l}\text { Rasgos redoximórficos distribuidos } \\
\text { en el perfil completo }\end{array}$ \\
\hline & & $9-25$ & $\mathrm{Bt}$ & $\mathrm{AL}$ & $10 \mathrm{YR} 3 / 2$ & $\bullet$ & Fir & MP, Ad & Presencia de agua inter-agregados \\
\hline & & $25-45$ & $\mathrm{Cg} 1$ & FAAr & $5 \mathrm{Y} 4 / 1$ & $\bullet$ & Fri & P, LAd & $\begin{array}{l}\text { Rasgos redoximórficos } 50 \%(10 \mathrm{YR} \\
5 / 6) \bullet \text {, presencia de carbón. Sin } \\
\text { estructura (masiva) }\end{array}$ \\
\hline & & $45-66$ & $\mathrm{Cg} 2$ & FAAr & $2.5 \mathrm{Y} 5 / 4$ & $\bullet$ & Fir & $\mathrm{P}, \mathrm{Ad}$ & $\begin{array}{l}\text { Rasgos redoximórficos } 80 \% \text { (5YR } \\
5 / 8) \bullet . \text { Sin estructura (masiva) }\end{array}$ \\
\hline & & $>66$ & $\mathrm{Cr}$ & FAL & $2.5 \mathrm{Y} 6 / 2$ & $\bullet$ & Fir & $\mathrm{P}, \mathrm{Ad}$ & $\begin{array}{l}\text { Rasgos redoximórficos } 20 \% \\
(7.5 \text { YR 5/6) •. Sin estructura } \\
(\text { masiva) }\end{array}$ \\
\hline
\end{tabular}

${ }^{\top}$ Corresponde a la denominación de horizontes genéticos maestros. ${ }^{\ddagger}$ Clase textural al tacto. $\mathrm{Ar}=$ arenosa; $\mathrm{ArF}=$ areno francosa; $\mathrm{F}=\mathrm{franca} ; \mathrm{FL}=$ franco limosa; $\mathrm{FAr}=$ franco arenosa $\mathrm{FAL}=$ franco arcillo limosa; $\mathrm{L}=$ limo; $\mathrm{AL}=$ arcillo limosa $;$. $d=$ no determinado. ${ }^{\S}$ Determinada según contenido de agua en campo al momento de la descripción del perfil. Fri = friable; Fir = firme; Mfri = muy friable; Mfir = muy firme. " Determinada en condiciones de completa saturación. MP $=$ muy plástico; $\mathrm{P}=$ plástico; $\mathrm{LP}=$ ligeramente plástico; $\mathrm{LAd}=$ ligeramente adhesivo; $\mathrm{Ad}=$ adhesivo.

$S E=f(a+b+c+\cdots n) * z(h)$

donde: $S E$ : Servicio ecosistémico, $f$ : función (lineal, potencial o exponencial) que describe una propiedad del suelo, $a$ : carbono orgánico del suelo, $b$ : textura, $c$ : tipo de arcilla, $n$ : cualquier propiedad del suelo, $z(h)$ : profundidad de cada horizonte genético de suelo.
Así, un suelo (p. ej. PRA-G) puede estar prestando un servicio de provisión de alimentos y de hábitat para los organismos en superficie $(0-10 \mathrm{~cm})$, mientras que en profundidad $(40-50 \mathrm{~cm})$ se observó un nivel freático permanente, como consecuencia de la discontinuidad textural del suelo, lo que pone en evidencia el servicio de regulación hídrica del mismo (Cuadro 3). 


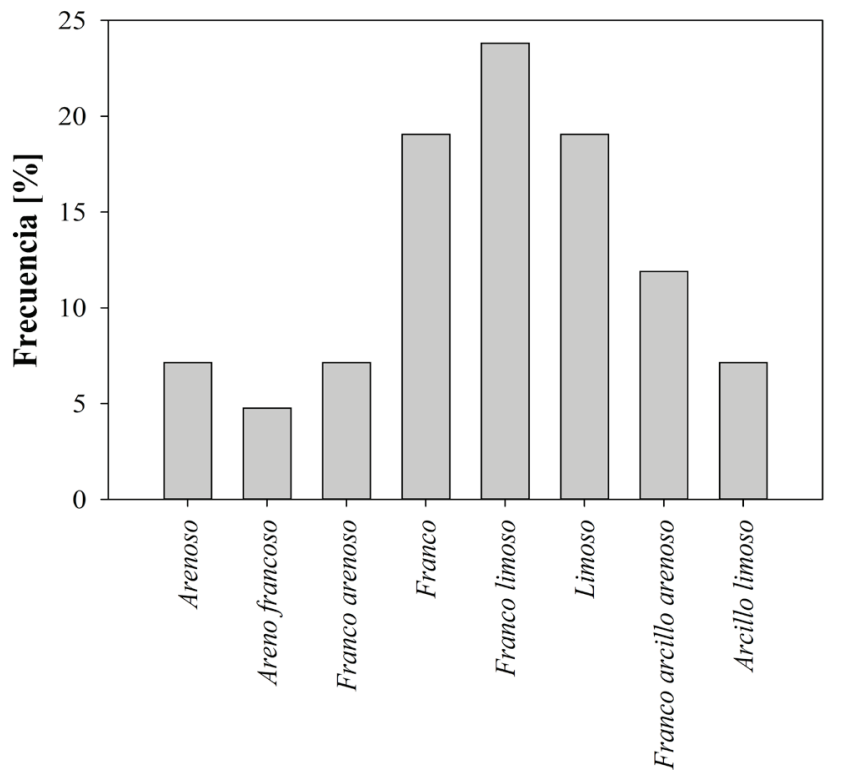

Clase textural

Figura 4. Distribución de frecuencia de clases texturales de 42 horizontes de suelos del departamento de Nariño, Colombia.

En términos generales, hay propiedades de los suelos que abarcan una mayor cantidad de funciones ecosistémicas como el carbono orgánico del suelo (Adhikari y Hartemink, 2016). Esta propiedad cumple un rol fundamental respecto a los servicios ecosistémicos que los suelos nos proveen (Lal, 2014). Franzluebbers (2010), indica que bajo sistemas de manejo conservacionistas (praderas y bosques) la materia orgánica muestra una estratificación, lo que se relaciona directamente con la calidad de suelo (Lal,

Cuadro 3. Servicios ecosistémicos de los suelos descritos en función de su uso actual.

\begin{tabular}{lcccc}
\hline \multirow{2}{*}{ Sitio } & \multicolumn{4}{c}{ Servicios ecosistémicos } \\
\cline { 2 - 5 } & Provisión & Regulación & Culturales & Soporte \\
\hline SUP & $*$ & $*$ & $*$ & $*$ \\
PAR & $*$ & $*$ & $*$ & $*$ \\
C-PAR & $*$ & $*$ & $*$ & $*$ \\
PRA-G & $*$ & $*$ & & $*$ \\
PAR-AZ & $*$ & $*$ & $*$ & $*$ \\
PRA-AZ & $*$ & $*$ & & $*$ \\
B-BOT & $*$ & $*$ & $*$ & $*$ \\
PRA-BOT & $*$ & & & $*$ \\
C-BOT & $*$ & & & $*$ \\
\hline
\end{tabular}

2014) y con funciones ecosistémicas claves como la producción de biomasa, el secuestro de carbono y la acumulación de agua (Adhikari y Hartemink, 2016).

La Figura 5 presenta la relación entre el contenido de carbono orgánico y la densidad aparente del suelo. Existe una sólida relación $\left(\mathrm{r}^{2}=0.83 ; P<0.0001\right)$ entre ambas variables que se describe a partir de una ecuación inversa de primer orden $(y=1 / \mathrm{a}+\mathrm{b} x)$, en donde: $y$ corresponde a la densidad aparente del suelo $\left(\mathrm{Mg} \mathrm{m}^{-3}\right)$ y $x$ al contenido de carbono orgánico total (\%).

A medida que incrementa el contenido de carbono, la densidad aparente disminuye. Esto es relevante porque a partir de esta FPT es posible inferir servicios ecosistémicos de regulación asociados a la densidad aparente, como la porosidad del suelo y su capacidad de almacenamiento de agua, que se vio reflejada en los sitios bajo páramo azonal (Figura 5).

No obstante en los usos de suelo en donde el contenido de carbono orgánico es una propiedad determinante (páramos y páramos azonales), ocurren otros servicios de provisión como pool genético (Lavelle et al., 2014) asociado a la diversidad de especies vegetales y animales; servicios de regulación como secuestro de carbono, purificación y regulación del agua o regulador de emisión de gases (p. ej. emisión de $\mathrm{CO}_{2}$ y $\mathrm{N}_{2} \mathrm{O}$ ); servicios culturales como recreación, inspiración, estética, sitios religiosos o de herencia cultural y finalmente servicios de soporte como formación de nuevo suelo a través de la meteorización o mineralización del material formador o como suelos proveedores de hábitat entre otros.

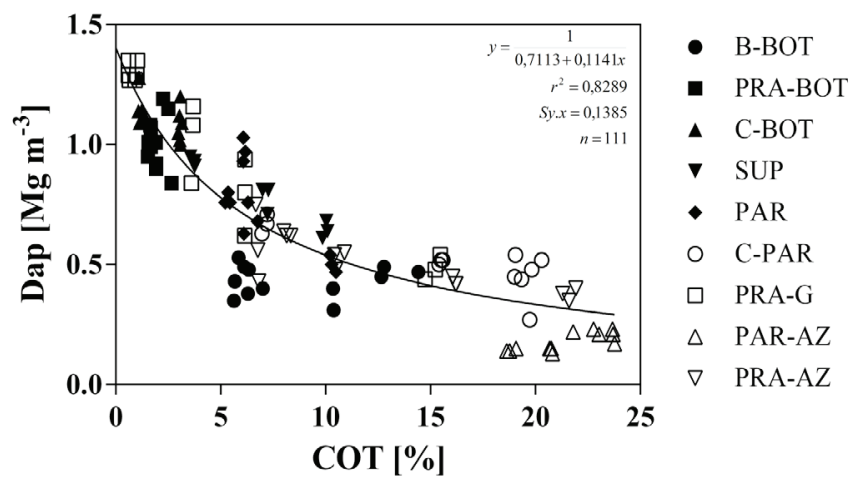

Figura 5. Función de pedotransferencia entre carbono orgánico (COT) y la densidad aparente (Dap) de los pedones evaluados. 
A medida que se intensifica el uso del suelo (praderas y cultivos) los SE se redistribuyen. En suelos bajo condición de praderas y cultivos, los SE de provisión (p. ej. producción de biomasa) toman protagonismo a expensas de una reducción de otras propiedades del suelo como el contenido de carbono orgánico (Figura 5). Por lo anterior, el desafío de la agricultura actual es poder mantener un equilibrio entre estos SE de provisión sin deteriorar los SE restantes, es decir, lograr mantener e incrementar la producción de alimentos con criterios de intensificación sostenible, donde el carbono orgánico del suelo puede ser utilizado como un indicador de este proceso (FAO, 2017b).

\section{Propuesta}

Durante el año 2015, en el marco de la celebración del Año Internacional del Suelo (FAO, 2015) se desarrollaron diversas iniciativas (ediciones especiales y congresos) para promover y proyectar el estudio de la ciencia del suelo (IUSS, 2015). Havlin et al.
(2010) indican que la Ciencia del Suelo ha encontrado un nicho de desarrollo en las ciencias ambientales, lo que pone de manifiesto la importancia del capital natural suelo y los servicios ecosistémicos que nos proveen. En ese contexto, y considerando que en ocasiones la presentación del suelo a la sociedad se realiza de manera abstracta, se proponen las "cartillas informativas de suelos" derivadas de los resultados de esta investigación (Figura 6A, 6B y 6C).

Estas cartillas no sólo representan el trabajo que realizan los científicos que describen y estudian las propiedades morfológicas del suelo, además vinculan el desarrollo de estos suelos a las condiciones de paisaje, otorgando una mirada geomorfológica del recurso. Otra función de estas cartillas es la divulgación de información, estas iniciativas pueden convertirse en una herramienta eficaz y didáctica para poder informar a la sociedad en su conjunto acerca de los suelos, de su desarrollo y de los diversos servicios ecosistémicos que el suelo nos provee, que ha permitido sustentarnos como humanidad y persistir en el tiempo.
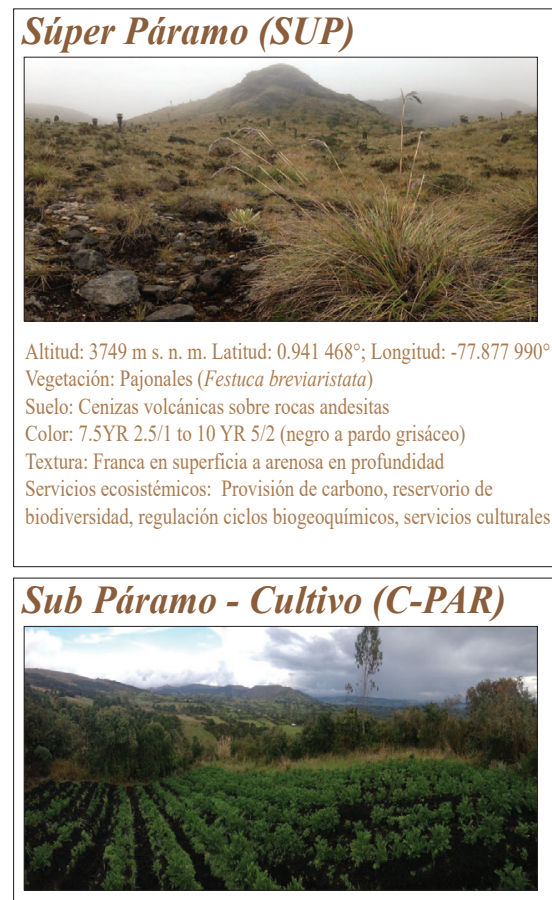

Altitud: $3402 \mathrm{~m} \mathrm{s.} \mathrm{n.} \mathrm{m.} \mathrm{Latitud:} 0.925494^{\circ}$; Longitud: -77.85530 Vegetación: Frailejón (Espeletia), pajonales (F. breviaristata), Cultivo de papa (Solanum tuberosum) Suelo: Cenizas volcánicas; Color: 10 YR 2/1 (negro) Textura: Limoso en superficie a franco arenoso en profundidad Estructura: Bloques subangulares a angulares Servicios ecosistémicos: Provisión de carbono, Servicios ceosistemicos Provision de carbono, reservorio de biodiversidad, regulación ciclos biogeoquímicos, provisión de biomasa
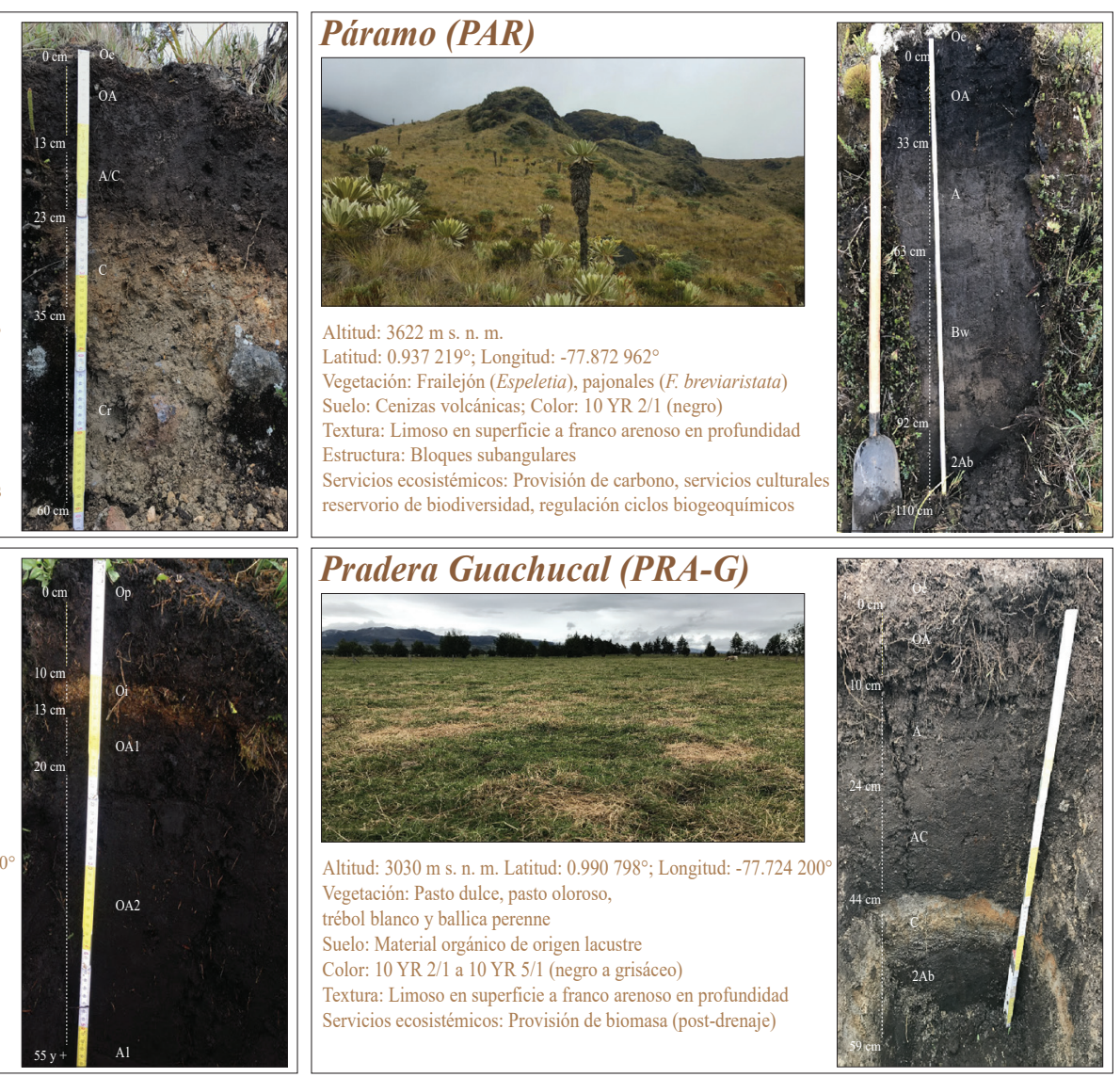

Figura 6A. Cartillas informativas de suelos y servicios ecosistémicos para el sitio de Cumbal. 


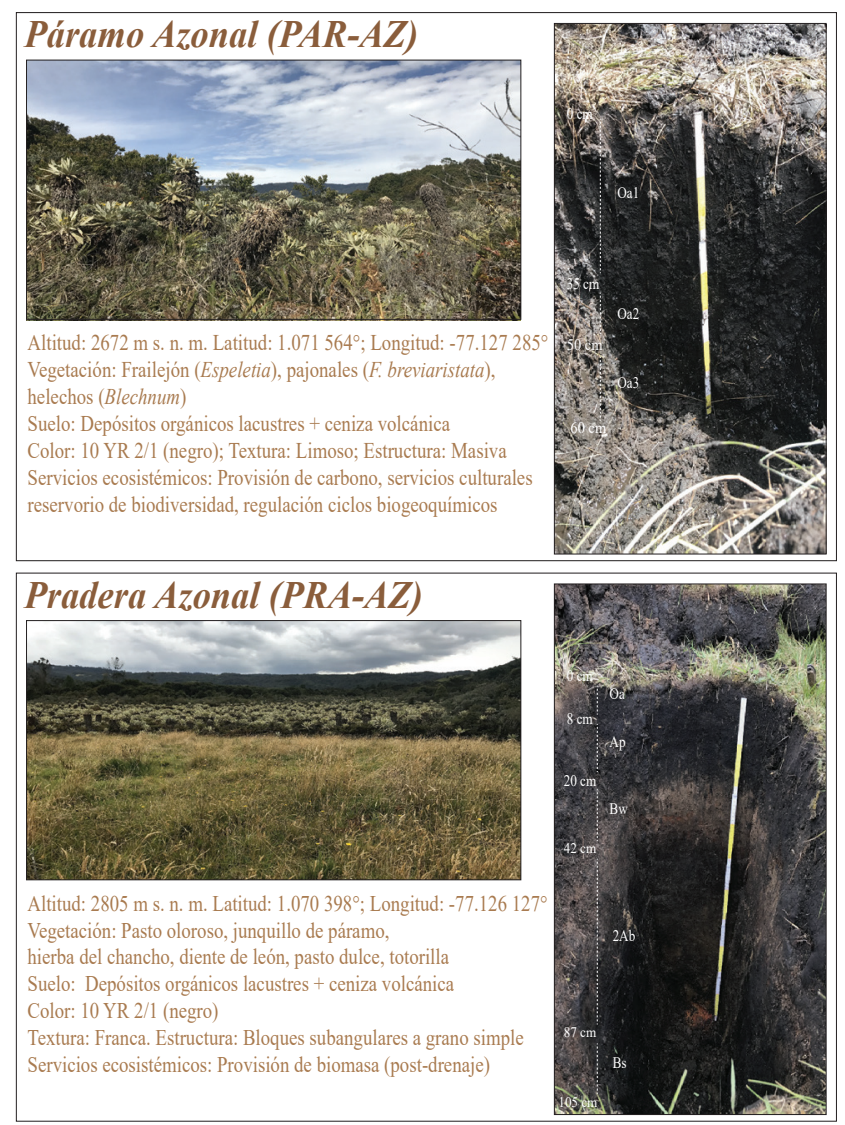

Figura 6B. Cartillas informativas de suelos $y$ servicios ecosistémicos para el sitio de El Encano.

\section{CONCLUSIONES}

- Las propiedades morfológicas del suelo se pueden vincular con propiedades funcionales. En ese sentido, el carbono orgánico del suelo fue utilizado como un estimador empírico de la densidad aparente de los suelos, parámetro que permite inferir servicios ecosistémicos de regulación de los Páramos de zona Andina. No obstante, es necesario profundizar en FPTs que permitan describir aspectos funcionales del sistema poroso de estos suelos.

- La información recolectada se convirtió en una herramienta eficaz de instrucción ambiental para la función que deben cumplir cada uno de los usos evaluados en la regulación de servicios ecosistémicos, con especial prioridad en los Páramos y Bosques de zona Andina de Nariño.

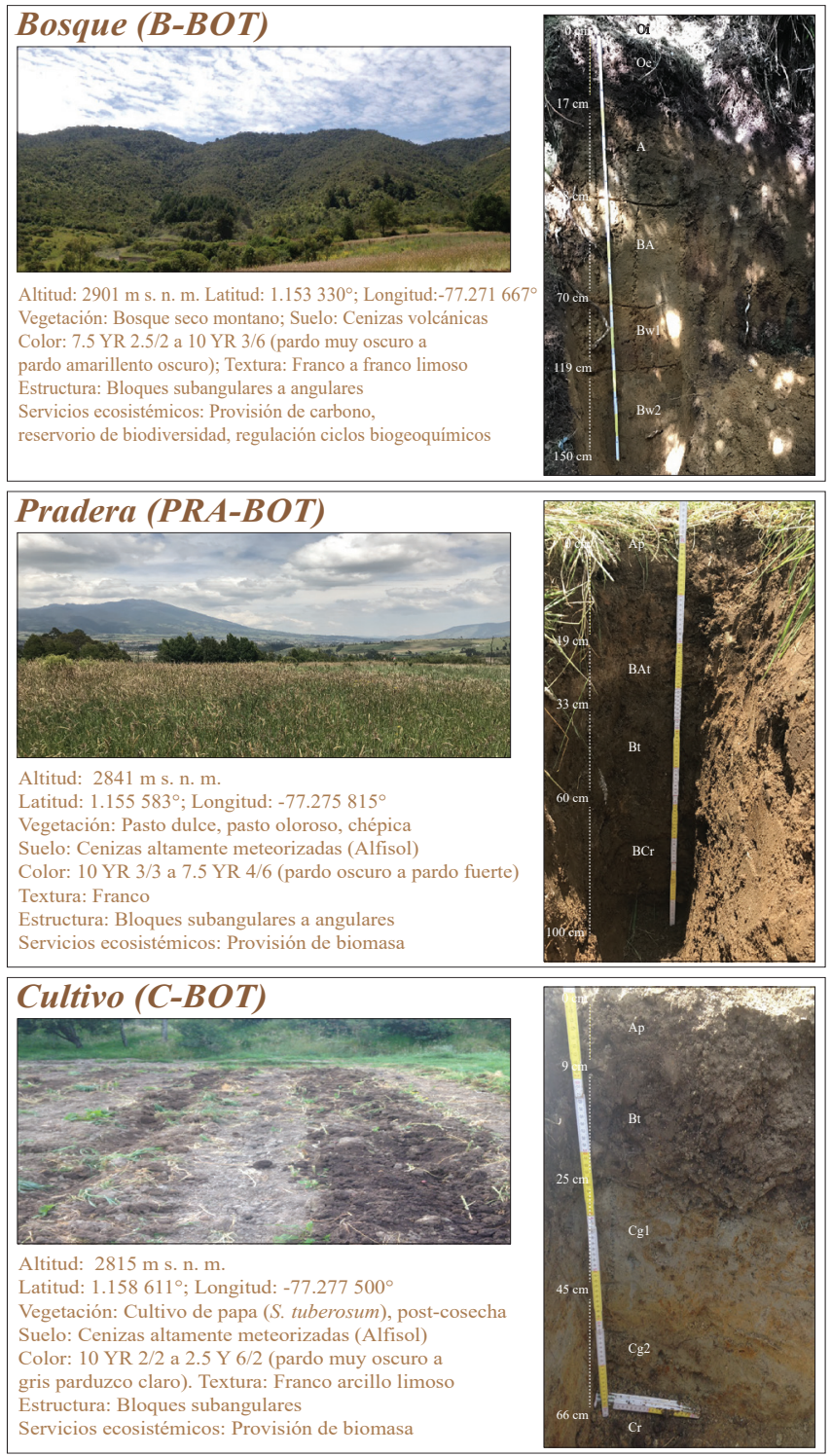

Figura 6C. Cartillas informativas de suelos y servicios ecosistémicos para el sitio de Botana.

\section{AGRADECIMIENTOS}

El primer autor agradece a la Plataforma de Movilidad Estudiantil y Académica de la Alianza del Pacífico, a la Vicerrectoría de Investigaciones, Postgrados y Relaciones Internacionales (VIPRI), Granja Experimental Botana y Laboratorios Especializados de la Universidad de Nariño, Viviana Bastidas, Daniela Bacca, Lesvy Ramos, Andrés "Mono" Rosero, Jairo Mosquera, Susana Valle, Nelson Narváez, Gabriela Quiroz y a la Reserva Natural "Encanto Andino" ubicada en la Laguna de La Cocha. 


\section{LITERATURA CITADA}

Adhikari, K. and A. E. Hartemink. 2016. Linking soils to ecosystem services - A global review. Geoderma 262: 101-111. doi: 10.1016/j.geoderma.2015.08.009.

Besoain, E. 1985. Mineralogía de arcillas de suelos. Bib. Orto IICA/CATIE. San José, Costa Rica.

Bockheim, J. G., A. N. Gennayidev, R. D. Hammer, and J. P. Tandarich. 2005. Historical development of key concepts in pedology. Geoderma 124: 23-36. doi: 10.1016/j. geoderma.2004.03.004.

Brevik, E. C. and A. E. Hartemink. 2010. Early soil knowledge and the birth and development of soil science. Catena 83: 23-33. doi: 10.1016/j.catena.2010.06.011.

Brussaard, L., P. C. De Ruiter, and G. G. Brown. 2007. Soil biodiversity for agricultural sustainability. Agr. Ecosyst. Environ. 121: 233-244. doi: 10.1016/j.agee.2006.12.013.

Burbano-Orjuela, H. 2016. El suelo y su relación con los servicios ecosistémicos y la seguridad alimentaria. Rev. Cienc. Agr. 33: 117-124. doi:10.22267/rcia.163302.58.

Canadell, J., R. B. Jackson, J. R. Ehleringer, H. A. Mooney, O. E. Sala, and E.-D. Schulze. 1996. Maximum rooting depth of vegetation types at the global scale. Oecologia 108: 583-595. doi: 10.1007/BF00329030.

Casanova, M., O. Seguel, A. Joel, I. Messing, W. Luzio y W. Vera. 2003. Funciones de pedotransferencia para conductividad hidráulica en laderas de secano. R.C. Suelo Nutr. Veg. 3: 42-48.

Costanza, R., R. D'Arge, R. de Groot, S. Farber, M. Grasso, B. Hannon, K. Limburg, S. Naeem, R. V. O’Neill, J. Paruelo, R. G. Raskin, P. Sutton, and M. van den Belt. 1997. The value of the world's ecosystem services and natural capital. Nature 387 : 253-260. doi: 10.1038/387253a0.

De Vos, B., B. Vandecasteele, J. Deckers, and B. Muys. 2005. Capability of loss-on-ignition as a predictor of total organic carbon in non-calcareous forest soils. Commun. Soil Sci. Plant Anal. 36: 2899-2921. doi: 10.1080/00103620500306080.

Dominati, E., M. Patterson, and A. Mackay. 2010. A framework for classifying and quantifying the natural capital and ecosystem services of soils. Ecol. Econ. 69: 1858-1868. doi:10.1016/j. ecolecon.2010.05.002.

Ellies, A., C. Ramírez, and R. Mac Donald. 2005. Organic matter and wetting capacity distribution in aggregates of Chilean soils. Catena 59: 69-78. doi: 10.1016/j.catena.2004.05.005.

FAO (Food and Agriculture Organization of the United Nations). 2015. Año Internacional del Suelo. Suelos sanos para una vida sana. http://www.fao.org/soils-2015/es/ (Consulta: agosto 10, 2017).

FAO (Food and Agriculture Organization of the United Nations). 2017a. Physical properties. http://www.fao.org/soils-portal/ soil-survey/soil-properties/physical-properties/en/ (Consulta: agosto 10, 2017).

FAO (Food and Agriculture Organization of the United Nations). 2017b. Alimentación y agricultura sostenibles. http://www. fao.org/sustainability/background/es/ (Consulta: agosto 10, 2017).
Franzluebbers, A. J. 2010. Depth distribution of soil organic carbon as a signature of soil quality. pp. 1-4. In: R. J. Gilkes and N. Prakongkep (eds.). Proceedings of the $19^{\text {th }}$ World Congress of Soil Science: Soil solutions for a changing world. Brisbane, Australia. 1-6 august 2010. http://iuss.org/19th\%20WCSS/ Symposium/pdf/2164.pdf

Grossman, R. B. and T. G. Reinsch. 2002. Bulk density and linear extensibility. pp. 201-228. In: J. M. Dane and G. C. Topp (eds.). Methods of soil analysis. Part 4. Physical Methods. SSSA Book Series, no. 5. Madison, WI, USA.

Hartemink, A. E. and J. G. Bockheim. 2013. Soil genesis and classification. Catena 104: 251-256. doi: 10.1016/j. catena.2012.12.001.

Havlin, J., N. Balster, S. Chapman, D. Ferris, T. Thompson, and T. Simth. 2010. Trends in soil science education and mployment. Soil Sci. Soc. Am. J. 74: 1429-1432. doi:10.213/ sssaj2010.0143.

Heimsath, A. M., W. E. Dietrich, K. Nishiizumi, and R. C. Finkel. 1999. Cosmogenic nuclides, topography, and the spatial variation of soil depth. Geomorphology 27: 151-172. doi: 10.1016/S0169-555X(98)00095-6.

Horn, R. and M. Kutilek. 2009. The intensity- capacity conceptHow far is it possible to predict intensity values with capacity parameters. Soil Tillage Res. 103: 1-3. doi: 10.1016/j. still.2008.10.007.

IUSS (International Union of Soil Science). 2015. Vienna Soil Declaration. "Soil matters for humans and ecosystems" Proclaimed at the Celebration of the International Year of Soils 2015 Achievements and Future Challenges. http://iuss. boku.ac.at/files/vienna_soil_declaration_december_7_.pdf (Consulta: abril 20, 2017).

Jenny, H. 1941. Factors of soil formation. A system of quantitative pedology. Dover. New York, NY, USA.

Krupenikov, I. A. 1993. History of soil science from its inception to the present. A. A. Balkema. Rotterdam, Netherlands.

Lal, R. 2004. Soil Carbon sequestration impacts on global climate change and food security. Science 304: 1623-1627. doi: 10.1126/science.1097396.

Lal, R. 2014. Soil conservation and ecosystem services. Int. Soil Water Conserv. Res. 2: 36-47. doi: 10.1016/S20956339(15)30021-6.

Lavelle, P., N. Rodríguez, O. Arguello, J. Bernal, C. Botero, P. Chaparro, Y. Gómez, A. Gutiérrez, M. P. Hurtado, S. Loaiza, S. X. Pullido, E. Rodríguez, C. Sanabria, E. Velásquez, and S. J. Fonte. 2014. Soil ecosystem services and land use in the rapidly changing Orinoco River Basin of Colombia. Agric. Ecosyst. Environ. 185: 106-117. doi:10.1016/j.agee.2013.12.020.

MEA (Millennium Ecosystem Assessment). 2005. Millennium ecosystem assessment: Ecosystems and human well-being synthesis. Island Press. Washington, DC, USA.

MINAMBIENTE (Ministerio de Ambiente y Desarrollo Sostenible). 2016. Política para la gestión sostenible del suelo. Ministerio de Medioambiente y Desarrollo Sostenible. Bogotá, D.C. Colombia. 
Ordoñez, M. C., L. Galicia, A. Figueroa, I. Bravo, and M. Peña. 2015. Effects of peasant and indigenous soil management practices on the biogeochemical properties and carbon storage services of Andean soils of Colombia. Eur. J. Soil Biol. 71: 28-36. doi: 10.1016/j.ejsobi.2015.10.001.

Pachepsky, Y. A., W. J. Rawls, and H. S. Lin. 2006. Hydropedology and pedotransfer functions. Geoderma 131: 308-316. doi: 10.1016/j.geoderma.2005.03.012.

Pryor, F. L. 1985. The invention of the plow. Comp. Stud. Soc. Hist. 27: 727-743.

Sadzawka R., A., M. A. Carrasco R., R. Grez Z., M. L. Mora G., H. Flores P. y A. Neaman. 2006. Métodos de análisis recomendados para los suelos de Chile. Revisión 2006. Instituto de Investigaciones Agropecuarias, Serie Actas INIA $\mathrm{N}^{\circ}$ 34. Santiago, Chile.

SAG (Servicio Agrícola y Ganadero). 2016. Pauta para estudio de suelos 2011 (Rectificada). SAG, Ministerio de Agricultura. Santiago, Chile.

Shoji, S., M. Nanzyo, and R. A. Dahlgren. 1993. Volcanic ash soils genesis, properties and utilization. Developments in Soil Science 21. Elsevier. Amsterdam, Netherlands.

Simonson, R. W. 1959. Outlines of a generalized theory of soil genesis. Soil Sci. Soc. Proc. 23: 152-156.

Schoeneberger, P. J., D. A. Wysocki, E. C. Benham, and Soil Survey Staff. 2012. Field book for describing and sampling soils, Version 3.0. Natural Resources Conservation Service, National Soil Survey Center. Lincoln, NE, USA.
Soil Survey Staff. 2014. Keys to Soil Taxonomy. USDA-NRCS. Washington, DC, USA.

UN (United Nations). Resolution adopted by the General Assembly on 20 December 2013. http://www.un.org/en/ ga/search/view_doc.asp?symbol=A/RES/68/232 (Consulta: abril 18, 2017).

USDA (United States Department of Agriculture). 1961. LandCapability Classification. Agriculture Handbook No. 210. Soil Conservation Service-USDA. Washington, DC, USA.

WAVES (Wealth Accounting and the Valuation of Ecosystem Services). 2016. WAVES Colombia Country Report 2016 Working Document. https://www.wavespartnership.org/en/ knowledge-center/waves-colombia-country-report-2016 (Consulta: abril 18, 2017).

WRB (World Reference Base for Soil Resources). 2014. International soil classification system for naming soils and creating legends for soil maps. World Soil Resources Reports 106. Food and Agriculture Organization of the United Nations. Rome, Italy.

Zúñiga, F., J. Dörner, O. Thiers y W. E. H. Blum. 2014a. ¿Qué nos quiere decir el Suelo? Agro Sur 42: 67-70. doi: 10.4206/ agrosur.2014.v42n2-08.

Zúñiga, F., D. Dec, S. R. Valle, J. Dörner y R. MacDonald. 2014b. Estabilidad estructural de un Andisol (Typic Durudand) bajo bosque nativo y pradera en el Sur de Chile. Agro Sur 42: 5566. doi: 10.4206/agrosur.2014.v42n3-06. 